\title{
Relaxation rate of excited electrons in metals: A nonperturbative calculation based on kinetic theory
}

\author{
I. Nagy, ${ }^{1,2}$ J. I. Juaristi, ${ }^{3}$ and P. M. Echenique ${ }^{3,4}$ \\ ${ }^{1}$ Department of Theoretical Physics, Institute of Physics, Technical University of Budapest, H-1521 Budapest, Hungary \\ ${ }^{2}$ Donostia International Physics Center, 20080 San Sebastián, Spain \\ ${ }^{3}$ Departamento de Física de Materiales, Facultad de Ciencias Químicas, Universidad del País Vasco, Aptdo. 1072, \\ 20080 San Sebastián, Spain \\ ${ }^{4}$ Centro Mixto CSIC-UPV/EHU, Aptdo. 1072, 20080 San Sebastián, Spain
}

(Received 30 June 2000; published 27 December 2000)

\begin{abstract}
By using an effective independent-particle model for an interacting electron gas, the relaxation rate of excited electrons close enough to the Fermi surface is calculated. The theoretical description is based on a binary collision treatment, implemented by an approximate nonlinear characterization of the residual screened interactions and exact phase-shift calculations. The important constituents of a consistent attempt are discussed. The results obtained are analyzed and compared to those based on perturbative linear-screening predictions. Reductions in the lifetime of excited electrons are found. Comparisons with experimental data from different sources are made.
\end{abstract}

DOI: 10.1103/PhysRevB.63.035102

PACS number(s): 71.10.Ca, 72.15.Lh, 71.45.Gm

\section{INTRODUCTION}

One of the most important manifestations of electronelectron interaction in the conventional theory of a freeelectron gas is dielectric screening. The dielectric function itself is an exact mathematical property of the interacting system, but can only be calculated approximately. The random phase approximation (RPA) is a well-known example. It is, in fact, a generalized self-consistent procedure, which may be derived ${ }^{1}$ by making the linearized Hartree equations time dependent in the treatment of the influence of a given external field (the field of a moving, charged particle) on the system. Alternatively, the same dielectric function $\left[\epsilon_{R P A}(q, \omega)\right]$ appears for the screening of electron-electron interactions in a field-theoretical (diagrammatic) approach, by selecting a class of diagrams., ${ }^{2,3}$

The validity limit for the applicability of $\epsilon_{R P A}$ in calculations of physical, measurable (or strongly related) quantities is clearly visible when one needs the static $(\omega=0)$ residual interactions. Well-established examples are the screening problems of charged impurities, i.e., a slow antiproton (heavy, repulsive particle) or a thermalized positron (light, attractive particle) in a free-electron system. In the case of strong interactions (in the practically important metallic range) between external probes and system electrons the assumption of a first-order Born approximation, i.e., the use of unperturbed (plane-wave) states for scattering final states, is not appropriate. For a heavy impurity one can apply density functional theory (DFT), which is a characteristic nonperturbative method of many-body theory. ${ }^{4-6}$ For a light impurity one can use a self-consistent, Kahana-type ${ }^{7}$ calculation, based on the Bethe-Goldstone formalism. ${ }^{8}$

Interestingly enough, in discussing the nature of the approximations inherent in a practical, perturbative implementation of the one-particle Green function method as applied to the electron gas, Hedin and Lundquist (building on the previously mentioned light-particle problem) already men- tioned the possible need to go beyond the RPA, in a consistent manner, to represent the screened electron-electron interaction. ${ }^{9}$ In the RPA, nonlinear effects in the representation of this interaction are completely neglected. ${ }^{10}$

These effects, in addition to their intrinsic conceptual interest, may have experimental importance and verification when the energy $(E)$ of an excited electron is close enough to the Fermi surface $\left(E_{F}\right)$ of the normal system. The coupling to the system, i.e., the relaxation process, is mediated by electron-hole excitations carrying energy $(\omega)$ and, due to the Pauli exclusion principle, $\max (\omega)=E-E_{F}$.

Theoretical study of the relaxation rate (inverse lifetime) is currently a very active field. ${ }^{11}$ Perturbative calculations, based on the electronic Bloch states (unperturbed states that are solutions of the same one-electron Hamiltonian) of a metal, have been performed recently ${ }^{12,13}$ within the standard one-particle Green function method. A detailed quantitative analysis of the nonlinear aspects by going beyond the (common) external-field concept along a consistent path is, therefore, desirable. This consistent path must include the following basic facts: the physical electrons are not infinitesimal charges; the system particles represent a charge distribution in a necessarily statistical many-body theory; their Coulomb hole is properly normalized, i.e., the maximum value of the hole density is constrained by the positive magnitude of the unperturbed density of the system.

The aim of the present paper is to obtain, in a consistent manner, theoretical values for the characteristic relaxation time $\tau$ of an excited electron interacting with a paramagnetic Fermi system of various (metallic) densities. In a comparative and deductive way we explore the role of some important ingredients of this theoretically interesting and experimentally verifiable many-body problem.

The paper is organized as follows. In Sec. II, we outline the kinetic model of the relaxation rate. The kinematical variables required in this treatment are fixed there. In Sec. III, we present detailed investigations of the proper form of a 
consistent screened potential, and of the role of applied scattering methods. The results are given analytically and numerically; illustrative figures are added. The last section, Sec. IV, is devoted to a comparison with selected experiments, and conclusions are given. We shall use Hartree atomic units, $e^{2}=\hbar=m_{e}=1$, throughout this work.

\section{KINETIC MODEL OF RELAXATION}

In the following we outline the description for the decay rate $(1 / \tau)$ of an excited electron. The decay is due to real scattering against the particles in the vicinity of the Fermi surface of a three-dimensional system. ${ }^{10}$ The system, at zero temperature, is characterized by its Wigner-Seitz radius $r_{s}$ determined from the homogeneous density $n_{0}$ via $r_{s}^{3}$ $=3 /\left(4 \pi n_{0}\right)$. We stress the point that we use an independentelectron picture for our normal system and the calculated electron-electron relaxation rate (inverse lifetime) is essentially determined (for a well-behaved screened interaction ${ }^{14}$ ) by the fundamental Pauli exclusion principle. ${ }^{15}$ Due to this principle, the kinetic model of decay remains a completely meaningful one for a Landau quasiparticle (a generic excited state of a normal system at its invariant Fermi surface ${ }^{16,17}$ ) if it behaves like an extra electron added to the cold Fermi sea. ${ }^{18}$

Here we shall calculate, thus implementing the kinetic model of the problem, the prefactor $\left[a\left(r_{s}\right)\right]$ of the expected expression for the relaxation rate: ${ }^{15}$

$$
\frac{1}{\tau}=a\left(r_{s}\right)\left(E-E_{F}\right)^{2} .
$$

In other words, we concentrate on the inclusion of basic facts and constraints (see the Introduction) in order to characterize the screened interactions required ${ }^{14}$ for the real scattering process, and follow a comparative method.

Since we are interested in decays close to the Fermi surface, all the colliding particles remain very close to this surface. ${ }^{10}$ The resulting decoupling (a characteristic feature of a degenerate system) of the angular and energy variables makes the calculation for $a\left(r_{s}\right)$ easier. Denoting by $\theta$ the usual scattering angle, and by $\alpha$ the angle between the two incoming momenta in the center-of-mass (c.m.) system, we can write ${ }^{19,20}$

$$
a\left(r_{s}\right)=\frac{1}{(2 \pi)^{4}}\left[\frac{w(\alpha, \theta)}{\cos (\alpha / 2)}\right]_{\mathrm{av}},
$$

in which $w(\alpha, \theta)$ is the transition probability for the collision process. By energy conservation, the scattering energy $\left(E_{c}\right)$ in the c.m. system is determined via $\cos \alpha=1-\left(E_{c} / E_{F}\right)$.

Finally, the average (av) is defined as follows: ${ }^{20,21}$

$$
\left[\frac{w(\alpha, \theta)}{\cos (\alpha / 2)}\right]_{\mathrm{av}}=\frac{1}{2 \pi} \int_{0}^{\pi} d \theta \int_{0}^{\pi} d \alpha \sin \alpha \frac{w(\alpha, \theta)}{\cos (\alpha / 2)} .
$$

In the knowledge of a screened (regular) potential one can formulate $w(\alpha, \theta)$ in terms of stationary scattering amplitudes by using the partial-wave expansion or Born approximation for these.

\section{SCATTERING POTENTIALS AND RESULTS}

Now, we are in the position to close our theoretical framework in the spirit of the Introduction and the previous section. In this way we shall calculate, based on Eqs. (2) and (3), first-order Born results for a given (in momentum space) screened potential $V(q)$.

First, we connect the present framework with the perturbative one-particle Green function method ${ }^{9}$ for the lifetime in an electron gas. We can write for the first-order Born transition probability

$$
w(\alpha, \theta)=2 \pi\left[\frac{2 \pi}{\mu} f(q)\right]^{2},
$$

in which $\mu=1 / 2$ is the reduced mass in the c.m. system and $f(q)=(\mu / 2 \pi) V(q)$ is the scattering amplitude (now, purely real). Using the peculiarity of the first-order Born approximation, i.e., $q=2 \sqrt{E_{c}} \sin (\theta / 2)=2 v_{F} \sin (\alpha / 2) \sin (\theta / 2)$ in our notation $\left(v_{F}^{2}=2 E_{F}\right)$, and furthermore a simple variable change $\theta \Rightarrow q$ in integrations, the twofold integrations in Eq. (3) result in a single integral for $a\left(r_{s}\right)$ :

$$
a\left(r_{s}\right)=\frac{2}{\pi v_{F}} \int_{0}^{2 v_{F}} d q[f(q)]^{2} .
$$

Note that this becomes precisely the well-known result of dielectric theory ${ }^{10,22,23}$ if we identify, via $\epsilon_{R P A}(q)$, the form of $V(q)$ as $V(q)=\left(4 \pi / q^{2}\right) / \epsilon_{R P A}(q)$.

Within the first-order Born approximation, it is customary to deduce analytical results by using a simple Yukawa-type potential $V(q)=4 \pi /\left(q^{2}+\beta^{2}\right)$, where $\beta^{2}=\left(4 v_{F}\right) / \pi$ if we take the Thomas-Fermi limit $(q \rightarrow 0)$ in $\epsilon_{R P A}(q)$. From Eq. (5) one obtains

$$
a\left(r_{s}\right)=\frac{1}{\pi v_{F}} \frac{1}{\beta^{3}}\left(\arctan \gamma+\frac{\gamma}{\gamma^{2}+1}\right),
$$

where the abbreviation $\gamma=\left(2 v_{F}\right) / \beta$ is introduced. This perturbative expression already heralds the special sensitivity of $\tau$ to screening $(\beta)$. The formal $v_{F} \gg 1(\gamma \gg 1)$ mathematical limit $\left(r_{s}=1.92 / v_{F}\right)$ of Eq. (6) is usually taken (with the above Thomas-Fermi screening parameter) by experimenters and applied as a reference value:

$$
a\left(r_{s}\right)=0.068 \times r_{s}^{5 / 2} .
$$

Note, furthermore, that a second-order Born approximation for the Yukawa potential already requires the application of Eq. (3), and not Eq. (5), because the second-order Born amplitude depends separately on the previous $q$ and $E_{c} \cdot{ }^{24}$

Of course, a meaningful inclusion of scattering requires, instead of a perturbation expansion, the use of the proper transition probability of stationary scattering theory: ${ }^{25}$

$$
w(\alpha, \theta)=2 \pi\left(\frac{2 \pi}{\mu}\right)^{2}|f(\theta)|^{2},
$$

where $f\left(E_{c}, \theta\right) \equiv f(\theta)$ is the exact (nonrelativistic) scattering amplitude in the c.m. system. It is given by 


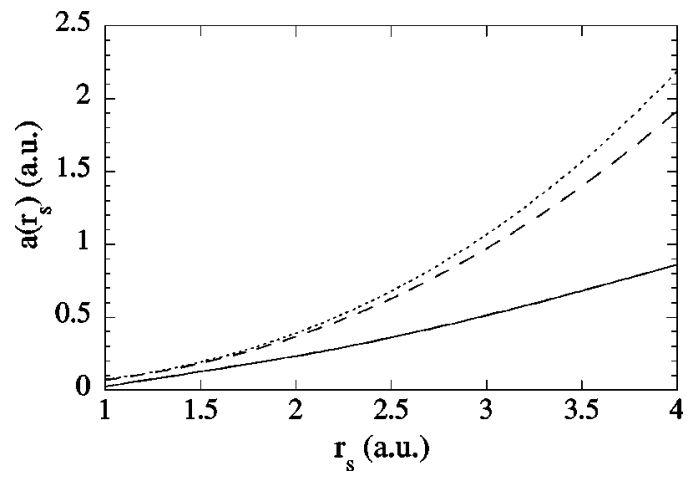

FIG. 1. The $a\left(r_{s}\right)$ functions of Eq. (1), obtained for the same linearized Thomas-Fermi potential. The solid curve corresponds to the phase-shift calculation. The dashed and dotted curves refer to the first-order Born approximation Eq. (6), and its high-density asymptotic form Eq. (7), respectively.

$$
f(\theta)=\frac{1}{\sqrt{E_{c}}} \sum_{l=0}^{\infty}(2 l+1) e^{i \delta_{l}} \sin \delta_{l} P_{l}(\cos \theta),
$$

and $\delta_{l}\left(E_{c}\right)$ are the usual phase shifts.

Next, we shall show the influence of the exact partialwave expansion on the relaxation rate by using the simple Yukawa potential $V(r)=(-Z / r) \exp (-\beta r)$, with $Z=-1$ and $\beta^{2}=\left(4 v_{F}\right) / \pi$. With this potential we perform numerical calculations to obtain phase shifts to Eqs. (8) and (9), and numerical integrations in Eq. (3). The accuracy of the numerical procedure was checked for the Born limit, Eq. (6), with the mathematical $Z \ll 1$ values. The result obtained for $a\left(r_{s}\right)$ is exhibited in Fig. 1 by a solid curve, together with those obtained from Eqs. (6) and (7). The latter are denoted by dashed and dotted curves, respectively. A simple comparison of perturbative and numerical results clearly signals the important role of a proper scattering treatment at metallic densities, even for a potential fixed a priori.

The next natural question concerns a physically reasonable form for the effective, residual interaction $V(r)$. The applicability of an RPA-based static screening $(\omega=0)$ has been discussed in many papers. Here we mention only a few important facts. It is well known from the pioneering work of Langer and Vosko $^{26}$ that the Coulomb hole around a negative charge is unphysical in the RPA. Although the hole itself is properly normalized (due to the use of the Poisson equation) its magnitude at the origin $[\delta n(0)]$ overestimates the background value $\left(n_{0}\right)$ for metallic densities. Quantitatively, $\delta n(0) / n_{0}=-2.2$ and $\delta n(0) / n_{0}=-6.4$, for $r_{s}=1.5$ and $r_{s}$ $=6$, respectively. The usual static RPA form of screening refers to a heavy impurity, i.e., the screening hole for a negative impurity is the self-consistent solution of linearized Kohn-Sham equations of DFT, treating them at the Hartree level. ${ }^{4}$ The mass of the probe particle is clearly missing in a RPA attempt. One can show this in the following way. The Kato cusp condition prescribes the proper derivative of configurational eigenfunctions or densities at the singularity ( $r$ $=0$ ) point of the Coulomb interaction. This condition is given by $^{27}$

$$
\left[\frac{n^{\prime}(r)}{n(r)}\right]_{r=0}=-2 Z \mu,
$$

in which $n(r)=n_{0}+\delta n(r), Z$ is the "impurity" charge, and the prime refers to derivation. This is an exact short-range condition. Furthermore, the derivative $\left[\delta n^{\prime}(0)\right]$ of the induced density is connected to its Fourier-space representation as $^{28}$

$$
\delta n^{\prime}(r=0)=-\frac{1}{8 \pi} \lim _{q \rightarrow \infty}\left[q^{4} \delta n(q)\right] .
$$

The application of the RPA for $\delta n(q)$ results in a factor of $-2 Z$ in Eq. (10), instead of $-2 Z \mu$, even at very high $\left[r_{s}\right.$ $\ll 1 ; n(r) \rightarrow n_{0}$ ] densities of the electronic system.

In order to satisfy these above-mentioned conditions, to allow the inclusion of the fact that our scattering pair consists of nonstatic particles, and to consider the necessarily statistical aspects of the many-body problem, we use a twoparameter, pseudononlinear treatment in the rest of this paper. Motivated by established verifications of this treatment for a slow ${ }^{29}$ and a swift ${ }^{30}$ antiproton, and for a thermalized positron, ${ }^{31}$ we approximate the hole density by the following expression:

$$
\delta n(q)=Z \frac{16 \pi n_{0}}{\lambda q^{4}+2 b q^{2}+16 \pi n_{0}},
$$

which is properly normalized in real space $(Z=-1$, in our case). The two parameters $\lambda$ and $b$ will be fixed below. The screened potential is calculated from the Poisson equation, and after inverse Fourier transformation we obtain

$$
\begin{aligned}
V(r)= & -\frac{Z}{r} \frac{1}{4 \alpha_{1} \alpha_{2}} e^{-\alpha_{1} r}\left[\left(\alpha_{1}+\alpha_{2}\right)^{2} e^{\alpha_{2} r}\right. \\
& \left.-\left(\alpha_{1}-\alpha_{2}\right)^{2} e^{-\alpha_{2} r}\right], \\
\delta n(r)= & \frac{Z}{r} \frac{n_{0}}{\lambda \alpha_{1} \alpha_{2}} e^{-\alpha_{1} r}\left(e^{\alpha_{2} r}-e^{-\alpha_{2} r}\right) .
\end{aligned}
$$

The screening parameters $\left(\alpha_{1}\right.$ and $\left.\alpha_{2}\right)$ are defined as follows:

$$
\begin{aligned}
& \alpha_{1}=\left[\frac{b}{2 \lambda}+\frac{\omega_{p}}{\sqrt{\lambda}}\right]^{1 / 2}, \\
& \alpha_{2}=\left[\frac{b}{2 \lambda}-\frac{\omega_{p}}{\sqrt{\lambda}}\right]^{1 / 2},
\end{aligned}
$$

where $\omega_{p}^{2}=4 \pi n_{0}$. Note the oscillatory behavior of $V(r)$ and $\delta n(r)$ for the complex case of $\alpha_{2}$. For a given $b\left(r_{s}\right)$, one can determine the nonlinearity parameter $\left[\lambda\left(r_{s}\right)\right]$ from the exact cusp condition, Eq. (10).

It may be of general interest to summarize the different, physically motivated parametrizations of Eq. (12). (A) With $\lambda=1$ and at high $q$ one gets the corresponding limit in the RPA. (B) With $b=2 v_{F}^{2} / 3$ and $\lambda=0$ one obtains the quasiclassical Thomas-Fermi result. (C) With $\lambda=1$ and $b=0$, one 


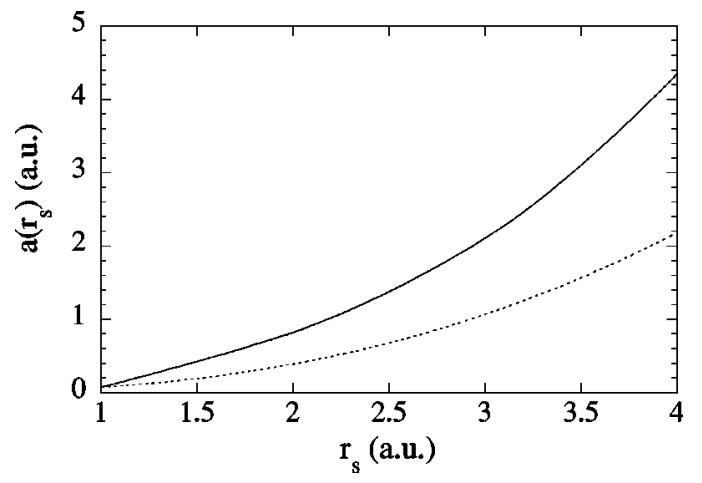

FIG. 2. Different $a\left(r_{s}\right)$ functions required in Eq. (1). The solid curve is based on nonlinear parametrizations (as a function of $r_{s}$ ) of Eq. (13), and phase-shift calculation for the scattering potentials. The dotted curve, as in Fig. 1, is the high-density first-order Born result, Eq. (7), obtained for a linearly screened potential.

can characterize the linear screening of a heavy particle in an ideal charged boson gas. ${ }^{32}$ (D) The $b=2 v_{F}^{2} / 3$ mentioned in point (B), of course, refers to an impurity situation. Its value for a dynamical situation, i.e., when both of the colliding partners are at the Fermi level, is expected to be higher, on physical grounds. In fact, by using a velocity $(v)$ dependent extension of screening ${ }^{30}$ for charged particles moving at $v$ $=v_{F}$, one gets the $b=2 \times\left(2 v_{F}^{2} / 3\right)$ result. (For earlier, confirming discussions of this effective enchancement, see Refs. 33 and 34.) In a recent paper on the physical consistency of the one-particle Green function method, Holm and von Barth also concluded ${ }^{35}$ that one of the most important questions is the proper treatment of screening for both the short- $(q$ $\rightarrow \infty)$ and long- $(q \rightarrow 0)$ range parts. Note that in a partially iterated calculation, i.e., beyond the free-electron propagator approximation but with RPA screening, they obtained similar results at $r_{s}=4$ to those given in our Fig. 1, although with somewhat smaller deviation [see their Fig. 10; $\Gamma \sim(1 / \tau)$ ].

We use the point (D) to fix $b\left(r_{s}\right)$ values in Eq. (12), and determine $\lambda\left(r_{s}\right)$ from the cusp condition. The potentials, Eq. (13), obtained in such a way are used to evaluate Eq. (8). The final resuls for $a\left(r_{s}\right)$ are plotted in Fig. 2, as a solid curve. The dotted curve in this figure refers to Eq. (7), i.e., to the perturbative result based on a linear-screening description. The deviations are notable, showing the nontrivial aspects of a consistent screening-scattering attempt. More generally, a strong nonlinearity indicates the greater difficulty in finding a diagrammatic expansion for it.

In order to get insight into the details of screening, in Fig. 3 we exhibit the results for the induced holes $\delta n(r)$ and corresponding screened potentials $V(r)$, at a particular value of the density parameter $r_{s}=2$. The solid curves in this figure refer to our consistent treatment [see point (D) and the text after it] and the dotted ones refer to simple, linearized Thomas-Fermi estimations. Due to the cusp condition, Eq. (10), our normalized Coulomb hole always satisfies the $|\delta n(0)| / n_{0}<1$ condition. In addition, and remarkably, our nonlinear potential is quite similar, in its shape and magnitude, to that of Pines. ${ }^{36}$ The latter (plotted by a dashed curve for the important range of $r$ ) was based on a sophisticated

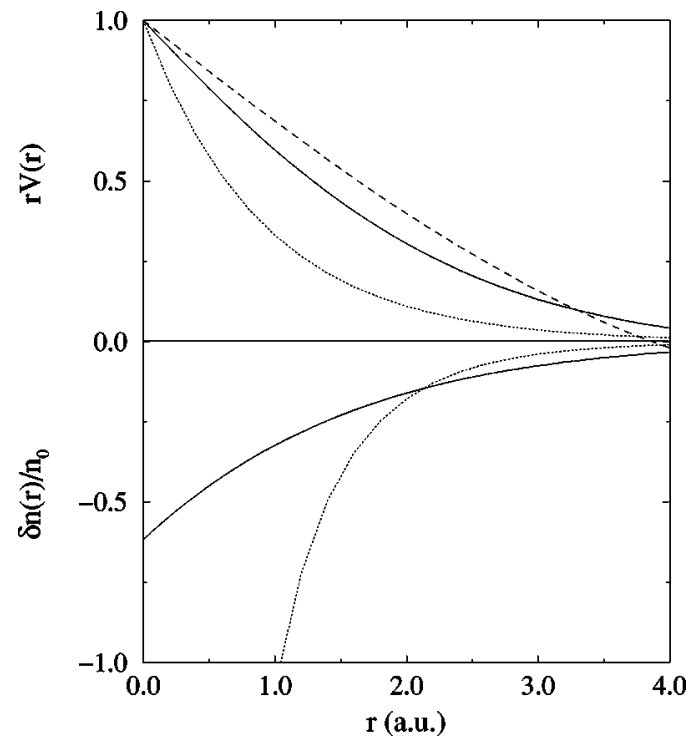

FIG. 3. United representation of induced Coulomb hole densities [normalized as $\delta n(r) / n_{0}$ ] and corresponding screened potentials [plotted as $r V(r)]$ for $r_{s}=2$. The solid curves are the results based on the nonlinear parametrization. The dotted curves are the results of a linearized Thomas-Fermi approximation. The dashed curve refers to the prediction of Pines for an effective screened interaction (see the text).

canonical transformation method for the interacting electron system, to characterize screened exchange and Coulomb correlation at metallic densities.

Note that our value for $1+\delta n(0) / n_{0}$ is smaller than the value obtained from the average pair correlation function $[g(r)]$ of the Hartree-Fock approximation $g(r=0)=1 / 2$. Physically, in the present nonlinear Hartree-type model the strong repulsion at short distances (determining the $\lambda$ ) and the average kinetic term (the $b$ factor) work against each other. At low densities $\left(r_{s} \gg 1\right)$ the Coulomb repulsion must win, and the screening should be similar to that of a constant-hole, i.e., Wigner-Seitz-like, picture. This latter, as the optimal electrostatic screening, may provide a bound for $a\left(r_{s}\right)$ in the dilute-fluid limit.

Finally, we close this section by calculating the relaxation rate for a light hole, which is constrained (by its momentum and energy) to the vicinity of the Fermi surface. Clearly, treating the hole as a moving positron we now have $b=2$ $\times\left(2 v_{F}^{2} / 3\right)$ and $Z=1$, in Eqs. (10), (12), and (13). We performed numerical integrations to obtain the phase shifts, and in Eq. (3) also. The hole-relaxation rates $\left[a\left(r_{s}\right)\right]$ obtained are plotted in Fig. 4, by the solid curve. The dotted curve refers to the electron-relaxation rates (solid curve in Fig. 2). By simple comparison one can deduce a remarkable "charge-sign" effect for the lifetime $\tau$. This effect is known, for heavy unit-charge intruders, as the Barkas effect. 6,30,37

\section{EXPERIMENTS AND CONCLUSIONS}

Within an effective independent-particle model, and based on equilibrium conditions of statistical physics, we deduced numerical values for the probability amplitudes 


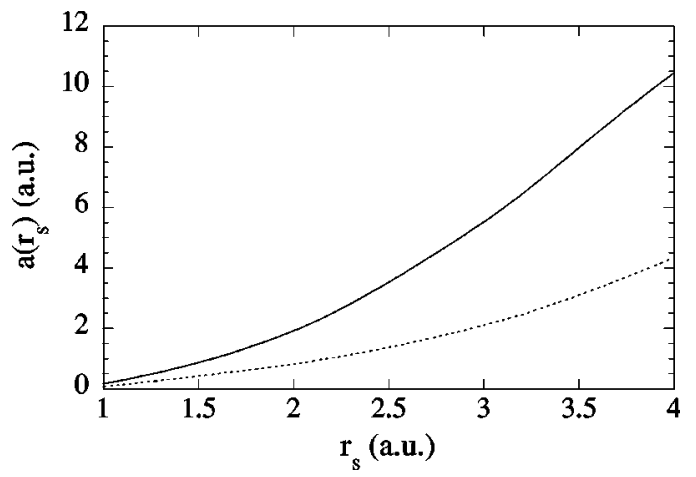

FIG. 4. Nonlinear results for the $a\left(r_{s}\right)$ function. The solid curve refers to the case of hole-electron scattering. For comparison the function for electron-electron scattering is plotted here by a dotted curve (solid curve in Fig. 2).

$[\exp (-t / \tau)]$ that determine the decay of distributions for particles prepared in given momentum-energy states close to the invariant Fermi surface. An interpretation of this decay as a finite lifetime implies an uncertainty in the one-particle energy. Therefore, the applicability limit (for the energy $E$ ) of our results is estimated, according to Pines and Nozieres, ${ }^{10}$ to be $(1 / \tau) \leqslant 2\left(E-E_{F}\right) / \hbar$, i.e., $a\left(r_{s}\right)\left(E-E_{F}\right) \leqslant 2$ in a.u. Excited electrons of this energy range have long enough lifetime and there is, in principle, a high probability for activating chemical reactions in the surface region of a metal. ${ }^{11,38}$ Of course, nonlifetime effects, i.e., due to impurities in a real target, may result in more complicated physics on the femtosecond time scale.

Due to the important developments in ultrafast laser technology for time-domain measurements, lifetimes of excited electrons have been and will be probed. ${ }^{38}$ These experiments are relevant for potential technological applications. Similarly, the method based on cold Scanning tunneling microscopy (STM) can be used ${ }^{39}$ to study the spatial decay of interference patterns; this process is governed by electronelectron scattering. The method is free from complicated cascade and depopulation effects, and thus it is close to the equilibrium condition, inherent in the theoretical attempts.

A recent experiment, ${ }^{40}$ performed by time-resolved twophoton photoemission for an $\mathrm{Al}$ target to obtain a relaxation time, indicates similar deviations from Eq. (7) as our theoretical curves show in Fig. 2. Further experiments for other free-electron-like metals (i.e., for $\mathrm{Mg}$ ) would help to understand the basic constituents of an especially sensitive theoretical quantity: the calculated lifetime of excited electrons. A recent analysis ${ }^{39}$ of a STM experiment for lifetimes at Ag and $\mathrm{Cu}$ surfaces shows the characteristic energy dependence and, in addition, reduction (about $70 \%$ ) of the lifetime in comparison with Eq. (7). An ingredient of the observed decay rate of electrons at metal surfaces arises from intraband transitions, as happens with hole states at the surface band according to a theoretical calculation. ${ }^{41}$ However, part of the above-mentioned reduction may also have its origin in nonlinearity.
The inelastic mean free path (IMFP) of electrons is an important parameter in photoelectron spectroscopy. It determines the sampling depth and is related to the instantaneous energy loss. ${ }^{42}$ The experimental data, based on synchrotron radiation, ${ }^{43}$ were investigated ${ }^{44}$ for the lighter alkali metals (Li: $r_{s}=3.3, \mathrm{Na}: r_{s}=4$ ) by using the perturbative treatment of the RPA. Below about $\left(E-E_{F}\right) \simeq 10 \mathrm{eV}$, a notable drop of experimental points below this theoretical estimation was found. An explanation for the drop, in our treatment, is the enhancement of $a\left(r_{s}\right)$ values (see Fig. 2). The IMFP is proportional $^{42}$ to $\left[a\left(r_{s}\right)\left(E-E_{F}\right)^{3}\right]^{-1}$, and the interactingelectron-gas model is expected to be valid for the experimental situation. ${ }^{45}$

A system of identical particles, not localized in space and in strong interaction with one another, represents to a genuinely important problem. The actual physical process, decay of the momentum distribution of excited states, is of great interest. ${ }^{11,38-40,43,46}$ In this paper we addressed this question by using some physically consistent approximations of many-body theory and stationary scattering formalism. The consistency is achieved by constructing a nonlinear effective scattering potential for electrons at the Fermi level and calculating the scattering amplitude beyond the common firstorder Born approximation. The influence of the chargedependent Coulomb correlations turned out to be important, and notable reductions in the lifetime of excited electrons were found. Future theoretical works, within nonperturbative attempts, may address the questions of exchange in scattering and screening, i.e., the role of spins of an embedded scattering pair, in a consistent way. The well-established ${ }^{47}$ spin-dependent effective potentials, obtained in linear response treatment with many-body local field methodology beyond the RPA, are applicable in principle only in firstorder Born approximation ${ }^{48}$ for the singlet and triplet parts of the Mott-like differential cross section. Careful theoretical calculations ${ }^{49,50}$ performed in such a manner gave enchancements for the scattering rate (inverse lifetime) in comparison with the standard RPA-based prediction. Clearly, the nonlinear aspect of the spin-dependent Pauli correlations, in addition to the charge-dependent ones, may provide an interesting problem in the physical process examined. In this respect the local density approximation, inherent in the mentioned local field methodology, requires special attention and care at lower electron densities due to the obvious Wigner-Seitz-like nature of the direct screening hole. Finally, time-dependent (transient and memory) aspects, beyond the adiabatic picture, may give a further challenge.

\section{ACKNOWLEDGMENTS}

The work of one of us (I.N.) was supported by the OTKA (Grant Nos. T025019 and T029813). J.I.J. and P.M.E. acknowledge support from UPV/EHU and Eusko Jaurlaritza, Ministerio de Educación y Cultura (Grant No. PB97-0636), and Iberdrola S.A. We are indebted for useful discussions to E. V. Chulkov, J. J. Quinn, R. H. Ritchie, and V. M. Silkin. 
${ }^{1}$ J. Lindhard, K. Dan. Vidensk. Selsk. Mat. Fys. Medd. 28, No. 8 (1954); L. Hedin, J. Phys.: Condens. Matter 11, R489 (1999).

${ }^{2}$ A.L. Fetter and J.D. Walecka, Quantum Theory of Many-Particle Systems (McGraw-Hill, New York, 1971).

${ }^{3}$ J.C. Inkson, Many-Body Theory of Solids (Plenum, New York, 1984).

${ }^{4}$ A. Sjölander and M.J. Stott, Phys. Rev. B 5, 2109 (1972).

${ }^{5}$ P.M. Echenique, R.M. Nieminen, J.C. Ashley, and R.H. Ritchie, Phys. Rev. A 33, 897 (1986).

${ }^{6}$ I. Nagy, A. Arnau, P.M. Echenique, and E. Zaremba, Phys. Rev. B 40, 11983 (1989).

${ }^{7}$ S. Kahana, Phys. Rev. 129, 1622 (1963).

${ }^{8}$ H. Stachowiak, Phys. Rev. B 41, 12522 (1990).

${ }^{9}$ L. Hedin and S. Lundquist, Solid State Phys. 23, 1 (1969).

${ }^{10}$ D. Pines and P. Nozières, The Theory of Quantum Liquids (Benjamin, New York, 1966).

${ }^{11}$ P.M. Echenique, J.M. Pitarke, E.V. Chulkov, and A. Rubio, Chem. Phys. 251, 1 (2000).

${ }^{12}$ I. Campillo, J.M. Pitarke, A. Rubio, E. Zarate, and P.M. Echenique, Phys. Rev. Lett. 83, 2230 (1999).

${ }^{13}$ W.D. Schöne, R. Keyling, M. Bandic, and W. Ekard, Phys. Rev. B 60, 8616 (1999).

${ }^{14}$ J.M. Luttinger, Phys. Rev. 121, 942 (1961).

${ }^{15}$ N.W. Ashcroft and N.D. Mermin, Solid State Physics (Saunders, Philadelphia, 1981).

${ }^{16}$ J.M. Luttinger, Phys. Rev. 119, 1153 (1960).

${ }^{17}$ M. Oshikawa, Phys. Rev. Lett. 84, 3370 (2000).

${ }^{18}$ R.B. Laughlin, Rev. Mod. Phys. 71, 863 (1999).

${ }^{19}$ A.A. Abrikosov and I.M. Khalatnikov, Rep. Prog. Phys. 22, 329 (1959)

${ }^{20}$ P. Morel and P. Nozières, Phys. Rev. 126, 1909 (1962).

${ }^{21}$ H.H. Jensen, H. Smith, and J.W. Wilkins, Phys. Rev. 185, 323 (1969).

${ }^{22}$ J.J. Quinn and R.A. Ferrell, Phys. Rev. 112, 812 (1958).

${ }^{23}$ R.H. Ritchie, Phys. Rev. 114, 644 (1959).

${ }^{24} \mathrm{R}$. Newton, Scattering Theory of Waves and Particles (Springer, New York, 1982).

${ }^{25}$ L.I. Schiff, Quantum Mechanics (McGraw-Hill, New York, 1968).
${ }^{26}$ J.S. Langer and S.H. Vosko, J. Phys. Chem. Solids 12, 196 (1959).

${ }^{27}$ T. Kato, Pure Appl. Math. 10, 151 (1951); E. Steiner, J. Chem. Phys. 39, 2365 (1963).

${ }^{28}$ J.C. Kimball, Phys. Rev. A 7, 1648 (1977).

${ }^{29}$ I. Nagy, B. Apagyi, and K. Ladányi, Phys. Rev. A 42, 1806 (1990).

${ }^{30}$ I. Nagy and B. Apagyi, Phys. Rev. A 58, R1653 (1998).

${ }^{31}$ I. Nagy, B. Apagyi, and K. Ladányi, Solid State Commun. 86, 209 (1993).

${ }^{32}$ A.A. Kaparica and O. Hipolito, Phys. Rev. A 26, 2832 (1982).

${ }^{33}$ W.H. Young, Phys. Rev. 129, 2019 (1963).

${ }^{34}$ C.H. Leung, M.J. Stott, and C.O. Almbladh, Phys. Lett. 57A, 26 (1976).

${ }^{35}$ B. Holm and U. von Barth, Phys. Rev. B 57, 2108 (1998).

${ }^{36}$ D. Pines, Phys. Rev. 92, 626 (1953).

${ }^{37}$ J.M. Pitarke, R.H. Ritchie, and P.M. Echenique, Phys. Rev. B 56, 13883 (1995).

${ }^{38}$ M. Petek and S. Ogawa, Prog. Surf. Sci. 56, 239 (1998).

${ }^{39}$ L. Bürgi, O. Jeandupeux, H. Brune, and K. Kern, Phys. Rev. Lett. 82, 4516 (1999).

${ }^{40}$ M. Bauer, S. Pawlik, and M. Aeschliman, Proc. SPIE 3272, 201 (1998).

${ }^{41}$ J. Kliewer, R. Berndt, E.V. Chulkov, V.M. Silkin, P.M. Echenique, and S. Crampin, Science 288, 1399 (2000).

${ }^{42}$ J.J. Quinn, Phys. Rev. 126, 1453 (1962).

${ }^{43}$ G.K. Wertheim, D.M. Riffe, N.V. Smith, and P.H. Citrin, Phys. Rev. B 46, 1955 (1992).

${ }^{44}$ L.I. Johansson and B.E. Sernelius, Phys. Rev. B 50, 16817 (1994).

${ }^{45}$ H. Yasuhara, S. Yoshinaga, and M. Higuchi, Phys. Rev. Lett. 83, 3250 (1999).

${ }^{46}$ B. Gumhalter and H. Petek, Surf. Sci. 445, 195 (2000).

${ }^{47}$ C.A. Kukkonen and A.W. Overhauser, Phys. Rev. B 20, 550 (1979); C.F. Richardson and N.W. Ashcroft, ibid. 55, 15130 (1997).

${ }^{48}$ S. Yarlagadda and G.F. Giuliani, Phys. Rev. B 61, 12556 (2000).

${ }^{49}$ D.R. Penn, Phys. Rev. B 22, 2677 (1980).

${ }^{50}$ A.H. MacDonald and D.J.W. Geldart, Can. J. Phys. 60, 1016 (1982). 